Cahiers de la recherche sur les droits fondamentaux

$2 \mid 2003$

Les titulaires particuliers des droits fondamentaux

\title{
L'inapplicabilité de l'article 6, § 1 de la Convention européenne des droits de l'homme au contentieux des étrangers
}

Demba Ndiaye

\author{
CpenEdition \\ Journals \\ Édition électronique \\ URL : https://journals.openedition.org/crdf/7727 \\ DOI : $10.4000 /$ crdf. 7727 \\ ISSN : 2264-1246 \\ Éditeur \\ Presses universitaires de Caen \\ Édition imprimée \\ Date de publication : 1 janvier 2003 \\ Pagination : 93-98 \\ ISBN : 2-84133-205-5 \\ ISSN : $1634-8842$
}

Référence électronique

Demba Ndiaye, «L'inapplicabilité de l'article 6, § 1 de la Convention européenne des droits de l'homme au contentieux des étrangers ", Cahiers de la recherche sur les droits fondamentaux [En ligne], 2 | 2003, mis en ligne le 18 décembre 2020, consulté le 14 novembre 2022. URL : http:// journals.openedition.org/crdf/7727 ; DOI : https://doi.org/10.4000/crdf.7727 


\title{
L'inapplicabilité de l'article 6, § 1 de la Convention européenne des droits de l'homme au contentieux des étrangers
}

\author{
Demba NDIAYE \\ Doctorant en droit public \\ Université de Caen Basse-Normandie
}

Introduction

I. L'exclusion du contentieux des étrangers du champ d'application de l'article 6, § 1 de la Convention

A. La combinaison restrictive de l'article 6 , $\$ 1$ de la Convention à l'article $1^{\text {er }}$ du Protocole $n^{0} 7$ du 22 novembre 1984

B. Le contentieux des étrangers n'emporte pas contestation sur des droits ou obligations de caractère civil ni n'a trait au bien-fondé d'une accusation en matière pénale

II. La restriction des garanties procédurales accordées aux étrangers

A. La combinaison des critères d'invocabilité de la Convention et de l'article $1^{\text {er }}$ du Protocole $n^{0} 7$

B. L'absence de garanties procédurales pour certains étrangers

Conclusion

\section{Introduction}

Dans l'architecture de la Convention européenne des droits de l'homme, l'article 6 constitue une disposition capitale. Cette importance est liée à des raisons à la fois de principe et de pratique ${ }^{1}$.

La raison de principe tient à ce que la Convention rappelle dans son préambule la prééminence de l'État de droit, c'est-à-dire un État qui aménage au profit de ses justiciables des garanties pour assurer une réelle protection de leurs droits et libertés ${ }^{2}$. Les garanties procédurales consacrées par l'article $6, \$ 1$ sont, dans ce sens, consubstantielles à l'État de droit. La Cour souligne par ailleurs la «place éminente que le droit à un procès équitable occupe dans une société démocratique ${ }^{3}$. Autrement dit, la garantie du procès équitable est au cour

1. J.-C. Soyer et M. de Salvia, «L'article 6 », in La Convention européenne des droits de l'homme. Commentaire article par article, L.-E. Pettiti, E. Decaux, P.-H. Imbert (dir.), 2 éd., Paris, Economica, 1999, p. 240 sq.

2. Sur le rôle des garanties procédurales dans la protection des droits substantiels, voir F. Bernard et A. Berthe, «Les garanties procédurales en matière de reconduite à la frontière au regard de la $\mathrm{CEDH} », R T D H, 1997, \mathrm{p} .16$.

3. Affaire Airey c. Irlande, 9 octobre 1979, A, $n^{\circ} 32, \$ 24$. 
même des fondements de l'État de droit et de tout régime démocratique. Quant aux raisons d'ordre pratique, elles se rattachent à la volonté de la Cour de protéger non pas des droits théoriques ou illusoires mais concrets et effectifs, en s'appuyant sur les droits de sauvegarde, notamment le droit à un procès équitable, comme garants fonctionnels des droits substantiels. Ceci explique sans doute l'immense succès de cette disposition devant la Cour de Strasbourg ${ }^{4}$. Elle a suscité une importante jurisprudence sans pour autant qu'on puisse systématiser les critères de son applicabilité. C'est d'autant plus vrai que le raisonnement de la Cour n'a pas toujours été cohérent. L'affaire Maaouia en est une illustration.

En effet, la Commission s'était déjà prononcée pour l'inapplicabilité de l'article $6, \S 1$ au contentieux relatif à l'entrée, au séjour et à l'éloignement des étrangers ${ }^{5}$. Elle avait considéré qu'une décision relative à un tel contentieux n'impliquait pas une accusation en matière pénale ni ne concernait des droits ou obligations de caractère civil.

Statuant en Grande Chambre et pour la première fois, la Cour s'est ralliée à cette position dans une décision du 5 octobre 2000, Maaouia c. France ${ }^{6}$.

Le requérant, M. Maaouia, un Tunisien entré en France à l'âge de 22 ans et marié à une Française, avait été condamné par les juridictions françaises pour vol avec armes et violences volontaires. Le ministre de l'Intérieur prit alors, à son encontre, un arrêté d'expulsion en date du 8 août 1991. M. Maaouia ayant refusé de s'y plier, il fut condamné le 19 novembre 1992 à un an d'emprisonnement et à dix ans d'interdiction du territoire pour soustraction à l'exécution d'un arrêté d'expulsion. Le 14 février 1994 le Tribunal administratif de Nice prononça un jugement annulant l'arrêté d'expulsion du 8 août 1991. Fort de ce jugement, le requérant sollicita le 12 août 1994 le relèvement de l'interdiction du territoire en faisant valoir qu'il était marié à une Française et qu'il détenait un titre provisoire de séjour. Il l'obtint par un arrêt de la cour d'appel d'Aix-en-Provence en date du 26 janvier 1998. Le 30 décembre 1997, M. Maaouia saisit la Commission des droits de l'homme d'une requête qui fut transmise à la Cour le $1^{\text {er }}$ novembre 1998, date d'entrée en vigueur du Protocole $n^{\circ} 11$. Le requérant se plaignait de la durée excessive de la procédure en relèvement de l'interdiction du territoire français, soit plus de 3 ans et 5 mois. Selon le Gouvernement français la procédure en relèvement de l'interdiction du territoire ne concernait pas une contestation portant sur des droits de caractère civil ou ayant trait au bien-fondé d'une accusation pénale. Par conséquent, aucune violation de l'article 6 , $\$ 1$ ne pouvait être invoquée.

La Cour devait se prononcer sur l'applicabilité de l'article $6, \$ 1$ au cas d'espèce. Elle devait dire si M. Maa- ouia pouvait invoquer cette disposition dans le cadre de la procédure de relèvement de l'interdiction du territoire national devant le juge français. Elle a répondu par la négative, confirmant ainsi l'exclusion du contentieux des étrangers du champ d'application de l'article $6, \$ 1$ (I). Elle a, par la même occasion, limité considérablement les garanties procédurales accordées aux étrangers par les instances de Strasbourg (II).

\section{L'exclusion du contentieux des étrangers du champ d'application de l'article 6, § 1 de la Convention}

Les mesures relatives à l'entrée, au séjour et à l'éloignement des étrangers ne sont pas soumises à l'article 6, $\$ 1$ de la Convention. Autrement dit, l'étranger qui conteste ce type de mesures ne peut bénéficier des garanties procédurales consacrées par ladite disposition, à savoir le droit à un procès équitable. Pour l'affirmer, la Cour s'appuie sur une argumentation en deux temps, pour le moins discutable. Elle a tout d'abord combiné l'article 6 , $\$ 1$ de la Convention à l'article $1^{\mathrm{er}}$ du Protocole $\mathrm{n}^{\circ} 7$ pour en déduire que ce type de contentieux est exclu du champ d'application de l'article $6, \S_{1}(\mathrm{~A})$. Ensuite et au surplus, elle a confirmé que les décisions relatives à l'entrée, au séjour et à l'éloignement des étrangers n'emportent pas contestation sur des droits et obligations de caractère civil ni n'ont trait au bien-fondé d'une accusation en matière pénale (B).

\section{A. La combinaison restrictive de l'article 6, § 1 de la Convention à l'article 1er du Protocole n 7 du 22 novembre 1984}

La Cour commence par rappeler qu' « il faut interpréter les dispositions de la Convention en prenant en considération l'ensemble du système conventionnel, y compris les dispositions des protocoles ${ }^{7}$. Cette technique d'interprétation, qui consiste à éclairer ou compléter un texte à la lumière d'un autre texte, n'est pas inconnue de la Cour ${ }^{8}$. Elle a, à plusieurs reprises, opéré une combinaison des articles de la Convention entre eux, notamment les articles 8,12 et 13 , ainsi qu'avec d'autres textes conventionnels adoptés dans le cadre de l'ONU ou du Conseil de l'Europe. Aussi, l'utilisation de cette technique par la Cour n'est pas critiquable en elle-même. La déduction qu'elle en a faite l'est beaucoup plus. En effet, la Cour rappelle d'abord que l'article $1^{\text {er }}$ du Protocole $\mathrm{n}^{\circ} 7$ contient des garanties procédurales applicables en cas d'expulsion d'étrangers et que le préambule du même

\footnotetext{
4. Sur l'importance du contentieux de l'article 6, voir M. Fabre, «Le droit à un procès équitable », JCP, 1998, I, p. 157.

5. Voir par exemple Req. $n^{\circ} 7729 / 76$, Agee c. Royaume-Uni, 17 décembre 1976, DR, 7, p. 164 sq. ; Req. $n^{\circ} 7289 / 75$, X. et Y. c. Switzerland, 14 juillet 1977, $D R$, 9, p. 57 sq. ; Req. no 9285/ 81, X., Y., et Z. c. Royaume-Uni, 6 juillet 1982, DR, 29, p. 205 sq.

6. Req. $\mathrm{n}^{\circ} 39652 / 98$, Maaouia c. France, 5 octobre 2000.

7. Ibid., $\$ 36$.

8. Voir F. Ost, «Originalité des méthodes d'interprétation de la Cour européenne des droits de l'homme», in M. Delmas-Marty, Raisonner la raison d'État, Paris, PUF (Les voies du droit), 1989, p. 418.
} 
Protocole «se réfère à la nécessité de prendre de nouvelles mesures propres à assurer la garantie collective de certains droits et libertés par la Convention [...]». Ensuite, elle ajoute que, «de la lecture combinée [de la Convention et du Protocole $\mathrm{n}^{\circ} 7$ ], il ressort que les États étaient conscients que l'article 6-1 ne s'appliquait pas aux procédures d'expulsion d'étrangers et qu'ils souhaitaient prendre des mesures spécifiques dans ce domaine ${ }^{9}$. Autrement dit, selon la Cour, le fait pour les États membres d'adopter de nouvelles garanties spécifiques aux procédures d'expulsion des étrangers témoigne de leur volonté d'exclure ces procédures du champ d'application de l'article $6, \S 1$ de la Convention ${ }^{10}$.

Cette façon d'interpréter la Convention et ses Protocoles est critiquable à plus d'un titre. D'abord au regard du droit international général, la Cour a elle-même rappelé, à plusieurs reprises, que la Convention européenne des droits de l'homme doit être interprétée selon les règles de la Convention de Vienne sur le droit des traités du 23 mai 1969. Aux termes de cette Convention, les traités doivent être interprétés en fonction de leur "contexte », terme défini au $\$ 2$ de l'article 31 de ladite Convention comme «a) tout accord ayant rapport au traité et qui est intervenu entre toutes les parties à l'occasion de la conclusion du traité ; b) tout instrument établi par une ou plusieurs parties à l'occasion de la conclusion du traité et accepté par les autres en tant qu'instrument ayant rapport au traité ». En application de cette disposition conventionnelle, le Protocole $n^{\circ} 7$ ne saurait constituer une expression de la volonté authentique des États parties à la Convention de $1950^{11}$, puisqu'il ne lie pas tous les États membres à la Convention ${ }^{12}$. Ensuite, il est quelque peu hasardeux de voir dans les termes du préambule du Protocole $\mathrm{n}^{\circ} 7$ une volonté affichée des États signataires d'exclure le contentieux des étrangers du champ d'application de l'article $6, \$ 1$ de la Convention ${ }^{13}$. La Cour tente d'ailleurs de corroborer son interprétation en faisant appel au rapport explicatif relatif au Protocole ${ }^{14}$.

Pourtant, une disposition on ne peut plus claire du Protocole $\mathrm{n}^{\circ} 7$ nous renseigne davantage sur ses rapports avec la Convention. Il s'agit de l'article 7 intitulé « Relation avec la Convention » qui dispose que « les États parties considèrent les articles 1 à 6 du présent Protocole comme des articles additionnels à la Convention et tou- tes les dispositions de la Convention s'appliquent en conséquence». Cette formulation de l'article 7 laisse penser que les États membres n'avaient pas l'intention d'exclure expressément le contentieux des étrangers du champ d'application de l'article $6, \$ 1$ de la Convention mais qu'au contraire, ils s'étaient fixé comme objectif d' «ajouter» au texte de la Convention certaines garanties supplémentaires.

La deuxième branche de l'argumentation de la Cour, qui consiste à considérer que le contentieux des étrangers n'emporte pas contestation sur des droits ou obligations de caractère civil ni n'a trait au bien-fondé d'une accusation en matière pénale, est tout aussi discutable.

\section{B. Le contentieux des étrangers n'emporte pas contestation sur des droits ou obligations de caractère civil ni n'a trait au bien-fondé d'une accusation en matière pénale}

La Cour considère que «les décisions relatives à l'entrée, au séjour et à l'éloignement des étrangers n'emportent pas contestation sur des droits ou obligations de caractère civil du requérant ni n'ont trait au bien-fondé d'une accusation en matière pénale dirigée contre lui, au sens de l'article $6, \$ 1$ de la Convention ${ }^{15}$. Autrement dit, les critères matériels posés par l'article 6, $\$ 1$ de la Convention ne sont pas, en l'espèce, réunis.

L'article $6, \$ 1$ exige en effet que le litige à l'occasion duquel il est invoqué porte sur une contestation sur des droits ou obligations de caractère civil ou sur une accusation en matière pénale. Il s'agit, selon la Cour ${ }^{16}$, de «notions autonomes ${ }^{17}$ affranchies de toute qualification du droit interne. Les critères d'applicabilité de l'article $6, \$ 1$ sont donc composés d'une branche civile et d'une branche pénale.

Dans leur branche " civile», ces critères s'entendent comme l'existence entre les parties d'un différend véritable et sérieux qui porte sur des droits reconnus en droit interne ${ }^{18}$. Autrement dit, la contestation, qu'elle porte sur l'existence, l'étendue ou les modalités d'exercice d'un droit ou d'une obligation, doit susciter une procédure dont l'issue sera déterminante pour le droit ou l'obligation

9. Affaire Maaouia c. France, $\$ 36$.

10. Ibid., $\$ 37$.

11. H. Tigroudja, "L'inapplicabilité de l'article $6, \$ 1$ de la Convention à la procédure de relèvement d'une interdiction du territoire», $R T D H$, 2002, p. $433-462$.

12. Au 26 septembre 2002, 33 États sur les 44 qui composent le Conseil de l’Europe étaient liés par le Protocole $n^{\circ} 7$. Les 11 États qui ne sont pas liés se divisent en deux groupes: d'une part ceux qui n'ont ni signé ni ratifié (Royaume-Uni, Belgique, Principauté d'Andorre, Malte, Liechtenstein) et d'autre part ceux qui ont signé mais qui n’ont pas encore ratifié (Espagne, Turquie, Portugal, Pologne, Allemagne, Pays-Bas).

13. Le préambule du Protocole $n^{\circ} 7$ stipule : «Les États membres du Conseil de l’Europe, signataires du présent Protocole, résolus à prendre de nouvelles mesures propres à assurer la garantie collective de certains droits et libertés par la Convention de sauvegarde des droits de l'homme et des libertés fondamentales, signée à Rome le 4 novembre 1950 (ci-après dénommée "la Convention"), sont convenus de ce qui suit. »

14. Arrêt Maaouia, $\$ 36$.

15. Ibid., $\$ 40$.

16. Affaire König c. République fédérale d'Allemagne, 28 juin 1978, A, nº $27, \$ 88-89$; affaire Deweer c. Belgique, 27 février $1980, \mathrm{~A}, \mathrm{n}^{\circ} 35, \$ 42$.

17. Sur ce sujet voir F. Sudre, "Le recours aux notions autonomes ", in L'Interprétation de la Convention européenne des droits de l'homme, F. Sudre (dir.), Bruxelles, Bruylant, 1998, p. 93 sq.

18. J.-C. Soyer et M. de Salvia, «L'article 6 », p. 237 sq. 
litigieux. Encore faut-il que ce droit ou cette obligation soit reconnu par le droit interne puisque l'article $6, \$ 1$ ne leur assure par lui-même aucun contenu matériel ${ }^{19}$. Entendu dans ce sens, il est certain que le contentieux de l'entrée, du séjour et de l'éloignement des étrangers ne porte sur aucun droit ou obligation de caractère civil. En effet, il est admis en droit international un principe dérivé de la souveraineté des États selon lequel il n'existe pas de droit d'entrer ou de séjourner dans le territoire d'un État dont on n'est pas national. Néanmoins, une mesure relative à l'entrée, au séjour ou à l'éloignement d'un étranger peut avoir des conséquences directes sur ses droits civils, notamment sur sa vie privée et familiale. La Cour a d'ailleurs admis que la mesure d'interdiction du territoire, qui frappe M. Maaouia, a des conséquences importantes sur sa vie privée et familiale. Elle estime cependant que le caractère accessoire de cette atteinte fait que la procédure de relèvement de l'interdiction du territoire national n'entre pas dans le domaine des droits civils protégés par l'article $6, \$ 1$ de la Convention.

Le raisonnement est pour le moins spécieux. En effet la Cour considère que la procédure de relèvement de l'interdiction du territoire national n'est pas du domaine des droits civils protégés par l'article $6, \S 1$. Or la question était plutôt de savoir si, dans le cadre de cette procédure de relèvement, on pouvait porter atteinte au droit civil reconnu et protégé par l'article 8 de la Convention : le droit au respect de la vie familiale? L'analyse de la jurisprudence de la Cour montre qu'elle n'hésite pas à protéger par ricochet le droit au respect de la vie familiale d'étrangers faisant l'objet d'une mesure d'expulsion par exemple $^{20}$. Ainsi le raisonnement de la Cour dans sa branche « civile» consiste à dire que certes le droit pour les étrangers de mener une vie familiale est un droit civil au sens de l'article $6, \S 1$, mais ils ne peuvent invoquer cette disposition dans le cadre d'une procédure d'éloignement du territoire qui, dans ses conséquences, peut y porter une atteinte grave. Cette position est critiquable en ce qu'elle instaure une sorte de discrimination dans le degré de protection des droits en fonction de la nationalité des justiciables.

Dans sa branche pénale, le champ d'application matérielle de l'article 6, $\S 1$ se limite aux « accusations en matière pénale ». Il s'agit d'une notion autonome que la Cour interprète en s'appuyant largement sur le droit national notamment pour définir l'étendue de la «matière pénale ${ }^{21}$. Lorsque le système national qualifie un fait, ouvertement, d'infraction pénale, l'inscrit par exemple dans son code pénal, établit la compétence de ses juridictions pénales, les garanties de l'article $6, \$ 1$ doivent s'appliquer $^{22}$. La difficulté surgit lorsque, dans le droit national, la qualification pénale n'est pas consacrée. La Cour s'intéresse alors à la nature du fait ou du comportement transgresseur ainsi qu'au but et à la sévérité de la sanction pour déterminer s'il entre dans la matière pénale et par conséquent si l'article $6, \$ 1$ s'applique.

Dans le cas d'espèce, la Cour considère que la mesure d'interdiction du territoire ne porte pas davantage sur le bien-fondé d'une accusation en matière pénale. Pour s'en convaincre, elle invoque le caractère préventif de la mesure dans la plupart des États et le fait qu'elle puisse être prise par l'autorité administrative. On ne peut qu'être perplexe face à une telle argumentation, car, d'une part la Cour a dans le passé souligné à plusieurs reprises la dimension préventive et dissuasive du droit pénal ${ }^{23}$ et d'autre part, elle a déjà qualifié de pénales des sanctions prononcées par des autorités administratives ${ }^{24}$. Autrement dit, au regard même de la jurisprudence de la Cour, le caractère préventif d'une mesure et le fait qu'elle ait été prise par une autorité administrative ne suffisent pas à exclure les garanties de l'article $6, \S 1$. Il y va de la survie même de l'autonomie de la notion d'accusation pénale. Justifier l'inapplicabilité de cette disposition au contentieux de l'interdiction du territoire par le fait que cette mesure est préventive et qu'elle est prise par une autorité administrative est une rupture avec la jurisprudence constante de la Cour.

Que ce soit dans sa dimension civile ou pénale, le champ d'application de l'article $6, \$ 1$ fait l'objet d'une interprétation dynamique ${ }^{25}$ qui l'étend à des domaines plus ou moins inattendus ${ }^{26}$. Les étrangers échappent à cette dynamique, ce qui fait d'eux les justiciables les moins protégés dans le système européen de protection des droits de l'homme.

\section{La restriction des garanties procédurales accordées aux étrangers}

Les garanties procédurales reconnues aux étrangers par la Cour de Strasbourg sont restreintes d'abord parce qu'en application de la jurisprudence Maaouia, elles se

19. Affaire Pudas c. Suède, 27 octobre $1987, \mathrm{~A}, \mathrm{n}^{\circ} 125-\mathrm{A}, \S 30$.

20. Affaire Beldjoudi c. France, 26 mars 1992, A, nº 234-A à propos de l'expulsion d'un étranger divorcé dont l'enfant mineur, né en France, y est resté établi avec sa mère.

21. Selon la Cour, «si les États contractants pouvaient à leur guise qualifier une infraction de disciplinaire plutôt que de pénale [...], le jeu des clauses fondamentales des articles 6 et 7 se trouverait subordonné à leur volonté souveraine. Une latitude aussi étendue risquerait de conduire à des résultats incompatibles avec le but et l'objet de la Convention [...] », Affaire Engel et autres c. Pays-Bas, 8 juin 1976, A, $n^{\circ} 22, \$ 81$.

22. J.-C. Soyer et M. de Salvia, «L'article $6 »$, p. 254.

23. Affaire Malige c. France, 23 septembre 1998, JCP, 1999, II, $\mathrm{n}^{\circ} 10086, \S 34$.

24. Affaire Bendenoun c. France, 24 février 1994, A, nº 284, à propos des sanctions fiscales.

25. Sur l'interprétation dynamique des notions autonomes par la Cour, voir L. Favoreu et al., Droit des libertés fondamentales, Paris, Dalloz, 200o, p. 437 sq.

26. C'est ainsi que la Cour a considéré que le retrait de points sur un permis de conduire concerne une accusation pénale puisque d'une part la sanction intervient au terme d'une procédure pénale et qu'elle résulte donc de plein droit de la condamnation infligée par le juge pénal et d'autre part, parce que, s'agissant de la sévérité, le retrait de points peut entraîner l'annulation du permis de conduire: affaire Malige c. France, note F. Sudre. 
limitent aux seules garanties minimales de l'article $1^{\mathrm{er}} \mathrm{du}$ Protocole $n^{0} 7^{27}$. Ensuite, parce que, comme le constate le juge Costa dans son opinion partiellement concordante, la Cour a cru, à tort, devoir appliquer la règle du specialia generalibus derogant et cette erreur d'appréciation prive de toutes garanties procédurales une certaine catégorie d'étrangers (B) comme le démontre la combinaison normative des critères d'invocabilité de la Convention et de l'article $1^{\text {er }}$ du Protocole $n^{\circ} 7(A)$.

\section{A. La combinaison des critères d'invocabilité de la Convention et de l'article $1^{\text {er }}$ du Protocole $n^{0} 7$}

La Convention européenne des droits de l'homme, en son article $1^{\text {er }}$, dispose: «Les Hautes Parties contractantes reconnaissent à toute personne relevant de leur juridiction les droits et libertés définis au Titre I. » Autrement dit, toute personne justiciable d'un des États membres peut, en principe, bénéficier des droits du Titre I, donc du droit à un procès équitable sans aucune distinction de nationalité. Il suffit qu'un acte d'un État membre affecte une personne, fût-elle domiciliée en dehors du territoire de cet État ou n'y vivant pas, pour que la protection de la Convention opère ${ }^{28}$.

Il n'est pas nécessaire qu'existe un lien juridique stable comme la nationalité, la résidence ou le domicile pour que la protection de la Convention joue ${ }^{29}$. La précarité ou l'illégalité du séjour de la personne sur le territoire national n'a aucune incidence sur l'invocabilité de la Convention. C'est ce qui ressort de l'arrêt D. c. RoyaumeUni. Dans cette affaire, répondant à l'argument tiré de l'irrégularité du séjour du requérant, la Cour a précisé que le critère d'invocabilité de la Convention est la présence physique sur le territoire de l'État contractant, indépendamment de la qualification juridique de ce séjour de fait. Il suffit que l'étranger se trouve physiquement sur le territoire de l'État partie pour qu'il bénéficie de la protection de la Convention ${ }^{30}$.

La première incohérence fâcheuse de l'arrêt Maaouia est que la Convention, en sa qualité de lex generalis, s'adresse à toute personne y compris les étrangers en séjour régulier ou irrégulier, alors que l'article $1^{\mathrm{er}} \mathrm{du}$ Protocole $\mathrm{n}^{\circ} 7$, lex specialis censée y déroger, ne s'adresse qu'à une catégorie d'étrangers, ceux qui séjournent régulièrement sur le territoire d'un État contractant.
En effet, l'article $1^{\text {er }}$ du Protocole $n^{\circ} 7$ dispose : «Un étranger résidant régulièrement sur le territoire d'un État ne peut en être expulsé qu'en exécution d'une décision prise conformément à la loi et doit pouvoir : a) faire valoir les raisons qui militent contre son expulsion; b) faire examiner son cas; et c) se faire représenter à ces fins devant l'autorité compétente par une ou plusieurs personnes désignées par cette autorité [...]. » Cette disposition définit elle-même les limites de son invocabilité. Seul un étranger résidant "régulièrement» sur le territoire de l'État contractant est susceptible de bénéficier de ces garanties de procédure. Le terme "régulièrement» renvoie à la législation nationale de l'État qui est seul compétent pour déterminer les conditions de la régularité du séjour d'un étranger sur son territoire.

Il ressort ainsi de la combinaison des critères d'invocabilité de la Convention et de l'article $1^{\text {er }}$ du Protocole $\mathrm{n}^{\circ} 7$ que certaines catégories d'étrangers sont privées de garanties procédurales pour ce qui concerne les mesures d'éloignement du territoire national.

\section{B. L'absence de garanties procédurales pour certains étrangers}

La combinaison ainsi faite des critères d'applicabilité de la Convention et de l'article $1^{\text {er }}$ du Protocole $n^{\circ} 7$ met en évidence les limites du raisonnement de la Cour. Ainsi, certaines catégories d'étrangers ne bénéficient d'aucune garantie procédurale.

Il s'agit d'abord des étrangers qui se trouvent dans un État membre de la Convention non lié par le Protocole $n^{\circ} 7^{31}$. Le Protocole $n^{\circ} 7$ ne liant pas cet État, il ne peut être substitué aux garanties de l'article $6, \$ 1$ de la Convention celles de l'article $1^{\text {er }}$ dudit Protocole. Reste une question purement technique: peut-on juridiquement refuser à un étranger le bénéfice des garanties de l'article $6, \$ 1$ de la Convention alors que la lex specialis, censée s'y substituer, ne lie pas l'État défendeur? La question ne s'est pas encore posée telle quelle devant le juge européen mais la jurisprudence Maaouia y répond implicitement puisque qu'elle semble poser un principe absolu d'inapplicabilité de l'article $6, \S 1$ au contentieux des étrangers.

Une deuxième catégorie d'étrangers exclus des garanties de l'article $1^{\text {er }}$ du Protocole $n^{\circ} 7$ sont ceux qui se trouvent en situation irrégulière vis-à-vis de la législation

27. Certes les étrangers, au même titre que les nationaux, bénéficient d'autres garanties, notamment celles des articles 3 , 8 et 13 de la Convention. Ils bénéficient aussi de garanties procédurales accordées par le droit national. Mais contrairement à certains auteurs qui s'interrogent sur l'utilité d'un cumul des garanties procédurales nationales accordées aux étrangers avec celles de l'article 6 de la Convention (voir C. Teitgen-Colly, «L'éloignement des étrangers et les articles 5 et 6 de la CEDH ", in Les Étrangers et la Convention européenne de sauvegarde des droits de l'homme et des libertés fondamentales, H. Fulchiron (dir.), Paris, LGDJ, 1999, p. 307), nous pensons que les garanties de l'article 6 doivent être considérées comme un garant fonctionnel minimum pour assurer l'effectivité des droits substantiels contenus dans la Convention. Les États parties peuvent aller au-delà de ce minimum et accorder des garanties supplémentaires. Cependant, les multiples condamnations prononcées contre la France pour violation de l'article 6 prouvent que les garanties accordées par ce pays, aussi importantes soient-elles, ne suffisent pas à protéger les droits des justiciables.

28. S. Sarolea, «Les droits procéduraux du demandeur d'asile au sens des articles 6 et 13 de la Convention européenne des droits de l'homme », RTDH, 1999, p. 121.

29. J.A. Carrillo-Salcedo, «L'article $1^{\mathrm{er}}$ », in La Convention européenne des droits de l'homme. Commentaire article par article, p. 134 sq.

30. Affaire D. c. Royaume-Uni, 2 mai 1997, RDE, 1997, p. 94 sq.

31. Certains États comme l'Allemagne, la Belgique, l’Espagne, le Portugal ou le Royaume-Uni ne sont pas liés par le Protocole $n^{\circ} 7$. 
nationale d'un État partie à la Convention. En effet, alors qu'ils ne peuvent pas bénéficier des garanties procédurales de l'article $6, \$ 1$ en application de la jurisprudence Maaouia, l'article $1^{\text {er }}$ du Protocole $n^{0} 7$ ne leur est pas davantage applicable puisque cette disposition s'adresse uniquement aux étrangers en situation régulière ${ }^{32}$. Certes, la régularité du séjour est à apprécier selon la législation nationale. Mais d'autres instruments internationaux conclus dans le cadre du Conseil de l'Europe donnent quelques précisions sur la notion de "séjour régulier». Par exemple, la Convention européenne d'assistance sociale et médicale de 1953 dispose dans son article 11 que "le séjour est réputé irrégulier à dater de toute décision d'éloignement prise à l'encontre de [l'étranger], sauf s'il est sursis à l'exécution de cette mesure $»^{33}$. Autrement dit, dès lors que la personne a fait l'objet d'une décision d'expulsion, son séjour est considéré comme irrégulier, nonobstant les voies de recours contre une telle mesure du moment qu'elles ne sont pas suspensives.

Il en va de même de tous les étrangers entrés illégalement sur le territoire national et dont le séjour n'a pas été régularisé, ainsi que tous ceux qui se sont présentés à l'entrée du territoire (aéroports, ports et gares) et à qui on a refusé l'accès au territoire national. On peut, dès lors, constater avec évidence que ceux qui ne bénéficient d'aucune garantie procédurale au titre de la Convention ou de son Protocole $n^{\circ} 7$ sont les étrangers qui produisent le plus de contentieux en matière d'entrée, de séjour ou d'éloignement du territoire national.

\section{Conclusion}

Sir James Fawcett, président de l'ancienne Commission, délégué devant la Cour dans l'affaire König ${ }^{34}$, affirmait: "Selon moi, dans son interprétation de l'article 6, la Cour doit opérer des choix de politique judiciaire ${ }^{35}$. Plus de vingt ans après, la Cour vient d'adopter, sinon une jurisprudence politique, du moins une politique jurisprudentielle pour le moins discutable à l'égard des étrangers ${ }^{36}$. Elle a choisi de les priver tous des garanties procédurales de l'article $6, \$ 1$ de la Convention, sans leur garantir à tous la protection minimale de l'article $1^{\text {er }}$ du Protocole $n^{\circ} 7$. Il s'agit d'une jurisprudence qui s'inscrit sans doute dans une politique plus générale des gouvernements européens, caractérisée au niveau national par l'adoption de politiques migratoires restrictives et une limitation des garanties procédurales au profit des étrangers.

Aussi, les procédures en la matière se muent en des procédures d'exception, élaborées sur un mode préventif et souvent justifiées, sinon par une sorte de présomption sous-jacente de fraude ${ }^{37}$, du moins par une certaine méfiance envers cette catégorie de justiciables pas comme les autres. Les vœux du professeur Julien-Laferrière de voir le juge européen protéger davantage les droits des étrangers ${ }^{38}$ sont loin de se réaliser. Sans parler de déni de justice, on peut tout au moins s'interroger sur la pertinence d'un tel régime d'exception dans des États de droit.

32. Commission, affaire $n^{\circ} 20649$ / 92, S. T. c. France, 8 février 1993.

33. D. Lochak, «Protocole $\mathrm{n}^{\circ} 7$, article 1 », in La Convention européenne des droits de l'homme. Commentaire article par article, p. 1083 sq.

34. Affaire König, B, $\mathrm{n}^{\circ} 25$, p. 179.

35. Propos cités par le juge Loucaides dans son opinion dissidente sous l'arrêt Maaouia.

36. On peut regretter que cette décision, très discutable dans son fondement et lourde de conséquences pour les étrangers, n'ait ému que très peu de spécialistes.

37. S. Sarolea, «Les droits procéduraux... », p. 119.

38. F. Julien-Laferrière, «L'éloignement du territoire, des lois Pasqua et Debré à la loi Chevènement », in Les Étrangers et la Convention européenne..., p. 242. 